\title{
Increased serum protein levels by Yuanshi Shengmai Chenggu Tablet in treatment of avascular osteonecrosis of the femoral head
}

\author{
JIANCHUN ZENG, PENG DENG，JIE LI，WENJUN FENG，JINLUN CHEN and YIRONG ZENG
}

The Third Department of Orthopedics and Traumatology of Traditional Chinese Medicine, The First Affiliated Hospital of Guangzhou University of Traditional Chinese Medicine, Guangzhou, Guangdong 510405, P.R. China

Received October 19, 2016; Accepted June 13, 2017

DOI: $10.3892 / \mathrm{mmr} .2017 .8119$

\begin{abstract}
The traditional Chinese medicine (TCM) Yuanshi Shengmai Chenggu Tablet is used for treating the common orthopedic disease, hormone-induced avascular necrosis of the femoral head (ANFH) in China. However, its underlying mechanism and the changes induced in the treatment of ANFH remain to be fully elucidated. In the present study, through the use of isobaric Tag for Relative and Absolute Quantitation and multiple reaction monitoring quantifications, corticosteroid-induced femoral head necrosis and the effects of treatment with Yuanshi Shengmai Chenggu Tablet were examined. The aim was to identify serum proteins, which may be potential serum markers for the early clinical diagnosis of ANFH, and maybe used to develop more rapid and convenient detection strategies. A total of five proteins were identified, comprising Ig mu chain $\mathrm{C}$ region, keratin, type I cytoskeletal 9, properdin, apolipoprotein A-IV, and IQ and AAA domain-containing protein 1. The expression levels of all five proteins were lower in ANFH and were higher following TCM treatment. These findings were confirmed using ELISA and western blot analysis.
\end{abstract}

\section{Introduction}

Avascular necrosis of the femoral head (ANFH) is a common orthopedic disease (1-3) and is a medical problem for which no complete cure has been established $(4,5)$. ANFH is difficult to cure and its morbidity rate is high. Primary ANFH is predominately associated with specific genes in patients or due to gene mutation, whereas secondary ANFH includes traumatic and non-traumatic ANFH (6-8). It is well known that the etiology of traumatic ANFH is caused by the interruption of blood flow in blood vessels of the femoral head $(9,10)$. In previous years,

Correspondence to: Dr Yirong Zeng, The Third Department of Orthopedics and Traumatology of Traditional Chinese Medicine, The First Affiliated Hospital of Guangzhou University of Traditional Chinese Medicine, 16 Airport Road, Guangzhou, Guangdong 510405, P.R. China

E-mail: zeng6612@163.com

Key words: serum protein, traditional Chinese medicine, avascular osteonecrosis of the femoral head, biomarkers with the widespread clinical use of corticosteroids, cases of femoral head necrosis have increased substantially $(11,12)$. Although it has been demonstrated that the long-term use of a large dose of hormones and drinking are two important factors causing non-traumatic ANFH $(4,13)$, the detailed pathogenesis of non-traumatic ANFH remains to be elucidated. The Traditional Chinese medicine (TCM) Yuanshi Shengmai Chenggu Tablet is used for treating hormone-induced ANFH in China (14). Clinical investigations have shown that the application of this drug can significantly relieve pain in patients with ANFH, accelerate the absorption of necrotic bone and promote the formation of new bone, exhibiting relatively potent osteogenetic activity (14). However, the underlying mechanism and details of the changes induced by Yuanshi Shengmai Chenggu Tablet in the treatment of ANFH remain to be fully elucidated.

In previous decades, proteomics technology has developed rapidly and provides a high throughput, rapid and systematic analytical method for investigating biological processes and disease conditions. Clinical serum proteomics has become an active subject in modern science; particularly in investigations involving tumors, and it has provided important evidence for the early diagnosis and treatment of tumors (15). The application of proteomics on femoral head necrosis is relatively new; therefore, fewer reports are available.

In proteomics, the methods used to analyze large quantities of protein mixtures include the most commonly used isobaric Tag for Relative and Absolute Quantitation (iTRAQ) method, which is used with multiple reaction monitoring (MRM), and protein chip technology, which is used with surface enhanced laser desorption ionization time of flight mass spectrometry (SELDI-TOF MS). The iTRAQ method is used to separate proteins in mixtures through isoelectric points and molecular weights of proteins. This technology can be used to analyze the protein mixture in cells, tissues and serum, however, the isobaric Tag for iTRAQ has a low-resolution ratio in analyzing whole protein mixtures directly, and low abidance proteins may be missed. The resolution ratio can be improved by initial separation or purifying of samples, or pre-concentrating protein samples according to features $(15,16)$. The iTRAQ results are analyzed using software to identify the differential proteins. Following enzymolysis, analysis with MRM is performed. By combining the MRM result with the relevant database, the differential protein can be identified. 
The present study aimed to use ITRAQ and MRM to investigate corticosteroid-induced ANFH and the effects of treatment by Yuanshi Shengmai Chenggu Tablet, with a particular focus on the increased levels of serum proteins following treatment.

\section{Materials and methods}

Specimens. Serum from five patients with ANFH, who had been treated with a high-dose hormone (steroid) long term, and five patients treated with TCM (Yuanshi Shengmai Chenggu Tab) following steroid-induced ANFH, were prospectively collected at The First Affiliated Hospital of Guangzhou University of Traditional Chinese Medicine (Guangzhou, China), following the provision of written informed consent. The use of samples in the present study was approved by the Committees for Ethical Review of Research Involving Human Subjects of Guangzhou University of Traditional Chinese Medicine (Guangzhou, China). In addition, five healthy subjects were recruited during the same period, who were gender- and age-matched. The recruited population (average age: $39.46 \pm 5.32,50 \%$ male, $50 \%$ female) from February 2014 to February 2015 was divided into three groups: Steroid-induced ANFH (SANFH), SANFH-TCM treatment, and healthy controls. All blood samples were centrifuged at $1,250 \mathrm{x} \mathrm{g}$ for $5 \mathrm{~min}$ at room temperature and then $13,500 \mathrm{xg}$ for $15 \mathrm{~min}$ at $4^{\circ} \mathrm{C}$ within $1 \mathrm{~h}$ of collection. All samples were then stored at $-80^{\circ} \mathrm{C}$ until use.

iTRAQ analysis and MRM quantification. The iTRAQ labeling and MS analysis were performed as previously described (17). Subsequently, six iTRAQ-labeled sample polls were generated (two subgroups for each of SANFH, SANFH-TCM treatment and healthy controls). Briefly, high-abundance serum proteins, including albumin, IgG and haptoglobin, were removed using the Human 14 Multiple Affinity Removal system (Agilent Technologies, Inc., Santa Clara, CA, USA). Subsequently, $50 \mu \mathrm{g}$ protein from each sample was concentrated and desalted, followed digestion using trypsin prior to iTRAQ labeling. The six groups were labeled, including SANFH (iTRAQ reagent 113 and 116); SANFH-TCM treatment (iTRAQ reagent 114 and 117), and healthy controls (iTRAQ reagent 115 and 118). The six sample groups were mixed, desalted and dried.

The iTRAQ labeled peptides were separated using Strong Cation Exchange (SCX) chromatography. The SCX chromatography was performed on a polysulfoethyl $4.6 \times 100 \mathrm{~mm}$ column ( $5 \mu \mathrm{m}$; $200 \AA$; PolyLC Inc., Columbia, MA, USA). The peptides were eluted at the 45 min gradient from $100 \%$ buffer $\mathrm{A}$, containing $10 \mathrm{mM} \mathrm{KH}_{2} \mathrm{PO}_{4}(\mathrm{pH} \mathrm{3.0)}$ and $25 \%$ acetonitrile, to $45 \%$ buffer $\mathrm{B}$, containing $10 \mathrm{mMKH}_{2} \mathrm{PO}_{4}(\mathrm{pH} 3.0), 500 \mathrm{mM} \mathrm{KCl}$ and $25 \%$ acetonitrile, at the flow rate of $800 \mu \mathrm{l} / \mathrm{min}$ on an Agilent 1210 LC system. All fractions were analyzed using a matrix-assisted laser desorption/ionization-time of flight (TOF)/TOF 5800 mass spectrometer (AB SCIEX, Framingham, MA, USA). Protein quantification and identification were performed with the Proteome Discoverer (version 1.3; Thermo Fisher Scientific, Inc., Waltham, MA, USA). The default bias-correction was used and all quantitative variables were analyzed using Proteome Discoverer 1.3. Peptide abundances were calculated based on the areas of the monoisotopic peaks. Protein ratios were determined as the average ratios of all quantified peptides (18).
To validate the expression of biomarker candidates, MRM quantifications were performed as previously described (19). Briefly, $30 \mu \mathrm{g}$ protein of each sample were digested using trypsin prior to being desalted. The desalted peptide mixture was then loaded onto an Acclaim PePmap C18-reversed phase column (100 Á; Thermo Fisher Scientific, Inc.) and separated with a reversed phase C18 column (300 Á; Agela Technologies, Inc., Wilmington, DE, USA) mounted on a Dionex ultimate 3000 nano LC system. The peptides were eluted using a gradient of $5-80 \%(\mathrm{v} / \mathrm{v})$ acetonitrile in $0.1 \%$ formic acid over $45 \mathrm{~min}$ at a flow rate of $300 \mathrm{nl} / \mathrm{min}$, combined with a Q Exactive mass spectrometer (Thermo Fisher Scientific, Inc.). The eluates were then directly entered for Q-Exactive MS (Thermo, Fisher Scientific, Inc.), set in positive ion mode and a data-dependent manner, with a full MS scan between 350 and 2,000 m/z, a full scan resolution of 70,000 and an MS/MS scan resolution of 17,500. The MS/MS scan had a minimum signal threshold of $1 \mathrm{E}+5$ and isolation width of $2 \mathrm{Da}$. To evaluate the performance of this mass spectrometry on the iTRAQ-labeled samples, two MS/MS acquisition modes, higher collision energy dissociation (HCD), were used. To optimize the MS/MS acquisition efficiency of HCD, the normalized collision energy was systemically examined, stepped $20 \%$. Each MS/MS spectrum was searched against a mascot database (Uniprot_2015_human database; 20,194 protein entries, http://www.uniprot.org/taxonomy/9606) and a decoy database for false discovery rate (FDR) analysis (programmed in the software). The search parameters were as follows: Sample type, iTRAQ 8-plex (peptide-labeled); cysteine modification by methyl methane-thiosulfonate; digestion with trypsin enzyme; proteins with at least two peptides with a high confidence score ( $>95 \%)$ and a low FDR (estimated local FDR of 5\%); these were considered positively identified.

Western blot and ELISA assays. Protein extraction and blotting were performed as previously described (20). In brief, protein concentration was determined using a Protein Determination kit (Cayman Chemical, Ann Arbor, MI, USA). Subsequently, protein samples $(50-100 \mu \mathrm{g})$ were separated by $12 \%$ SDS-PAGE and electro-transferred to polyvinylidene fluoride membranes (Bio-Rad Laboratories, Inc., Hercules, CA, USA). The membranes were then incubated with 5\% non-fat skimmed milk in Tris-buffered saline containing $0.1 \%$ Tween 20 for $1 \mathrm{~h}$ at room temperature. Subsequently, the membranes were incubated overnight at $4^{\circ} \mathrm{C}$ with primary antibodies (anti-IgM Fab fragment, P01871; 1:1,000, sc-71330, Santa Cruz Biotechnology, Inc., Dallas, TX, USA; anti-Properdin, P27918; 1:1,000, sc-365664, Santa Cruz Biotechnology, Inc.; anti-Cytokeratin 9, P35527; 1:1,000, sc-58743, Santa Cruz Biotechnology, Inc.; anti-apoA-IV, P06727; 1:1,000, sc-374543, Santa Cruz Biotechnology, Inc.; anti-IQCA1, Q86XH1; 1:1,000, SAB1303425, Sigma-Aldrich; Merck KGaA, Darmstadt, Germany), followed by a subsequent incubation with horseradish peroxidase-conjugated secondary antibodies (goat anti-rabbit, cat. no. 12-348 and goat anti-mouse, cat. no. AP181P, Merck Millipore, Darmstadt, Germany) at $4^{\circ} \mathrm{C}$ for $1 \mathrm{~h}$. Densitometric quantification of the bands was conducted using ImageJ software (version 1.50; National Institutes of Health, Bethesda, MD, USA). The levels of Ig mu chain C region (IGHM; ab137982, Abcam, Cambridge, UK), properdin, keratin, type I cytoskeletal 9 (KRT9; ABIN417500, antibodies-online 
Normal

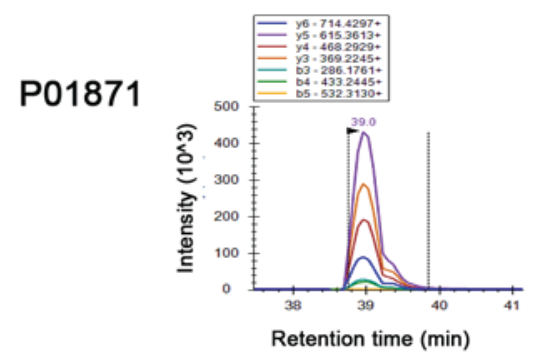

P27918

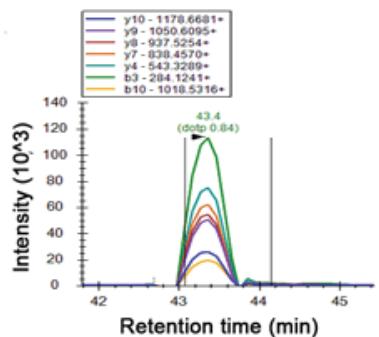

P35527

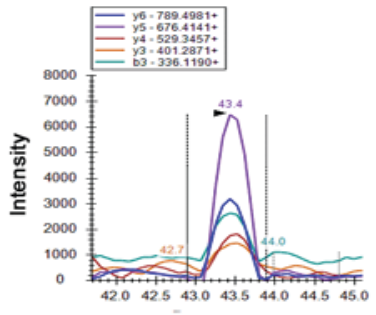

Retention time (min)

P06727
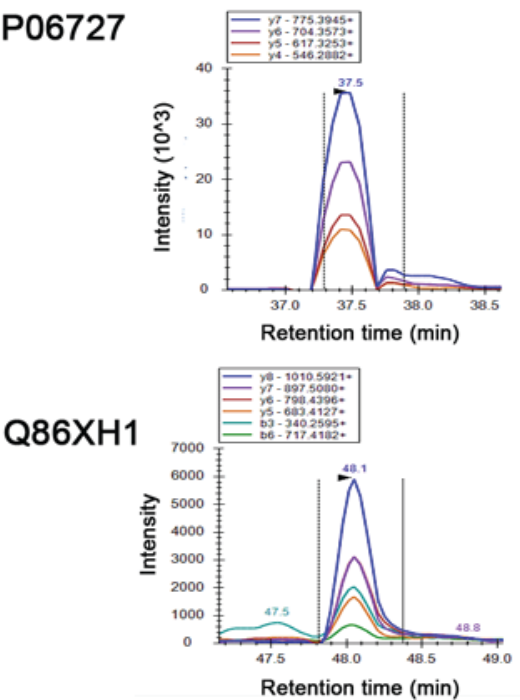

ANFH

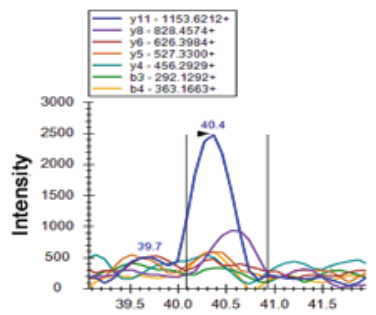

Retention time ( $\mathrm{min})$
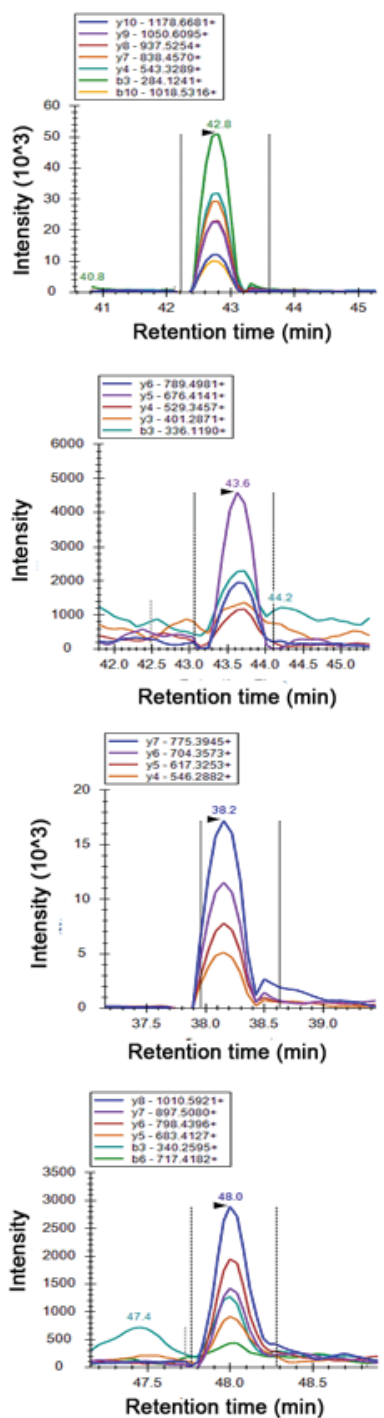

TCM

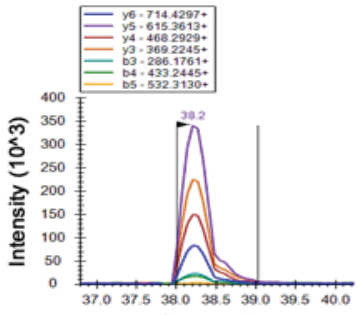

Retention time (min)
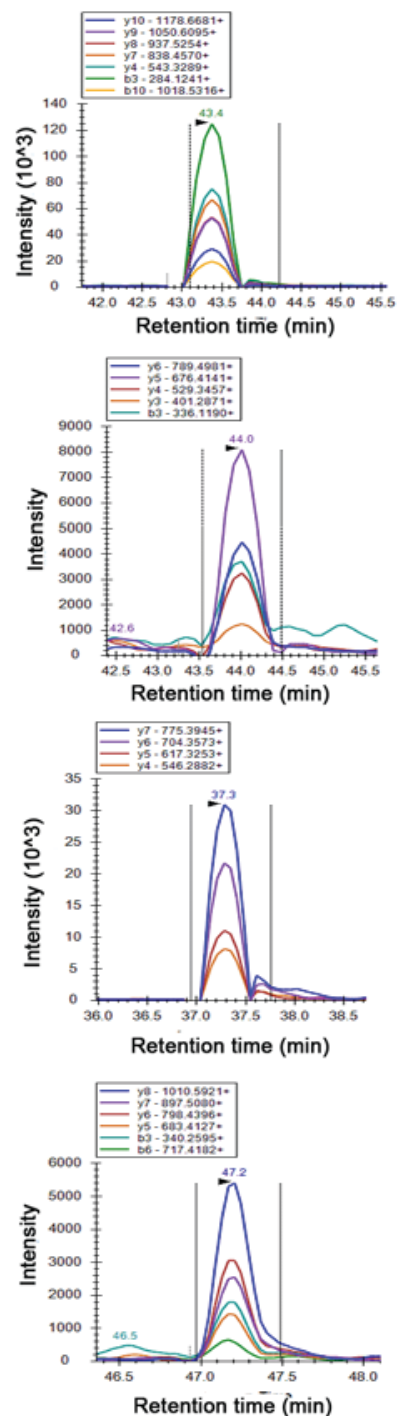

Figure 1. MRM quantification of serum proteins in normal subjects and in patients with ANFH +/-TCM treatment. ANFH, avascular necrosis of the femoral head; TCM, traditional Chinese medicine; P01871, Ig mu chain C region; P27918, properdin; P35527, keratin, type I cytoskeletal 9; P06727, apolipoprotein A-IV; Q86XH1, IQ and AAA domain-containing protein 1; b3, b4, b4, b5, b6, b10, y4, y5, y6, y7, y8, y9, y10 represent different peptide signals.

Inc., Atlanta, GA, USA), apolipoprotein A-IV (APOA4; ab214567, Abcam) and IQ and AAA domain-containing protein 1 (IQCA1; MBS9331190, MyBioSource, Inc., San Diego, CA, USA) were also quantified using ELISA kits according to the manufacturer's protocols.

Statistical analysis. All data were collected from at least three independent experiments and all results are expressed as the mean \pm standard deviation. Using the SPSS 20.0 software package (IBM SPSS, Armonk, NY, USA) (21), statistical analysis was performed using a Student t-test or one-way analysis of variance. $\mathrm{P}<0.05$ was considered to indicate a statistically significant difference.

\section{Results}

Levels of several serum proteins are decreased in ANFH, but increased following TCM treatment. In the present study, several serum proteins were identified, which were decreased in ANFH, but increased by TCM treatment, including P01871, 

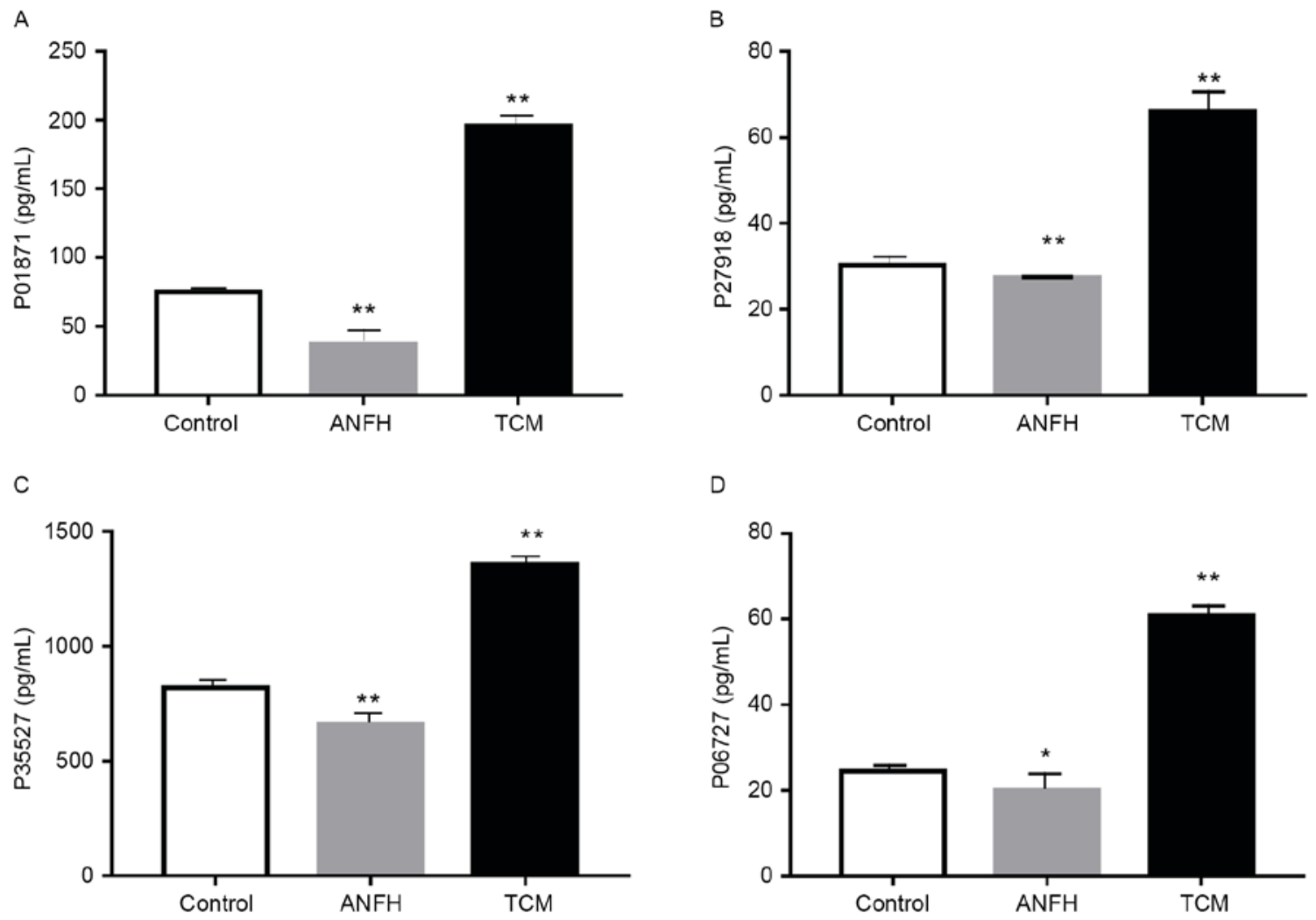

D
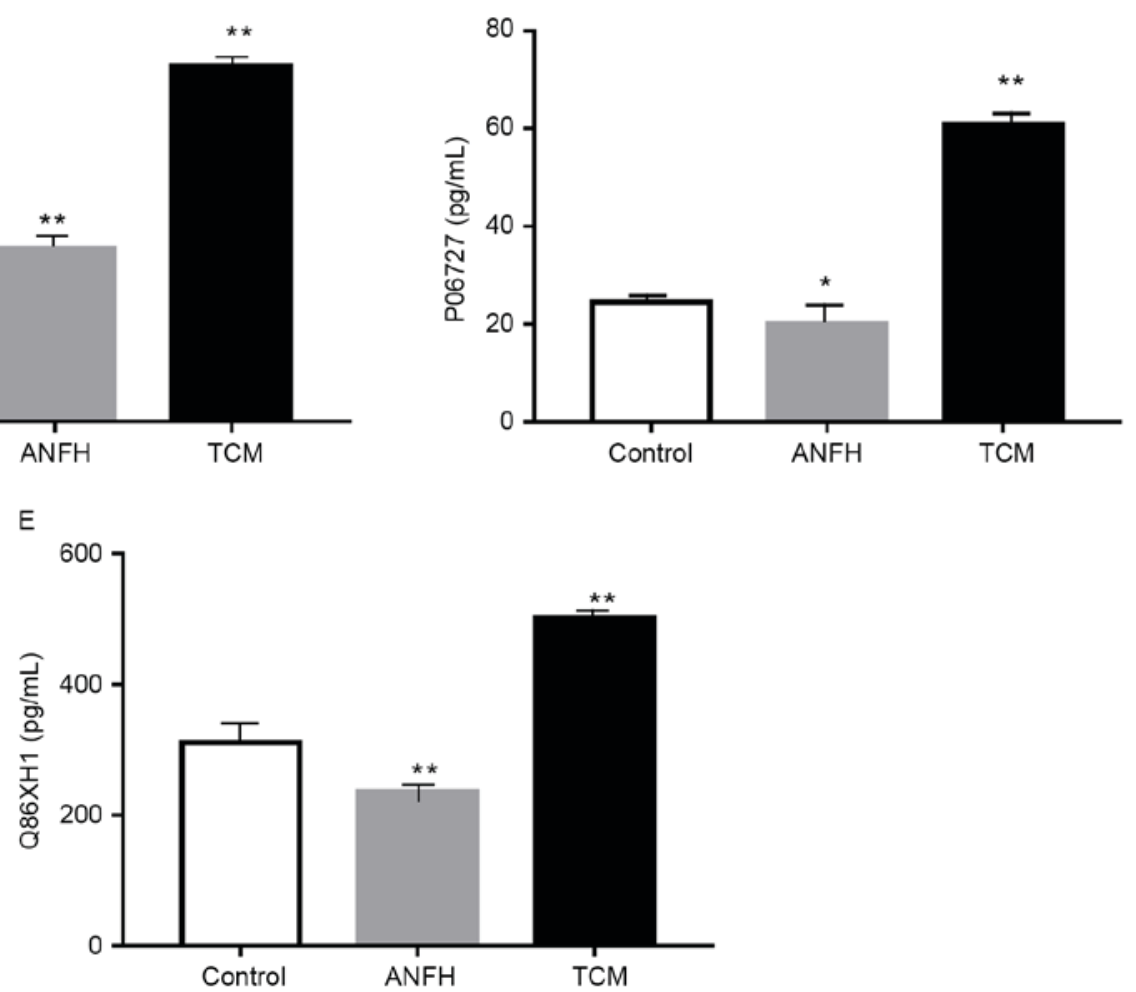

Figure 2. ELISA analysis of serum proteins in normal subjects and patients with ANFH+/- TCM. (A) P01871, (B) P27918, (C) P35527, (D) P06727, and (E) Q86XH1. "P<0.05 and "** $\mathrm{P}<0.01$ vs. control. ANFH, avascular necrosis of the femoral head; TCM, traditional Chinese medicine; P01871, Ig mu chain C region; P27918, properdin; P35527, keratin, type I cytoskeletal 9; P06727, apolipoprotein A-IV; Q86XH1, IQ and AAA domain-containing protein 1.

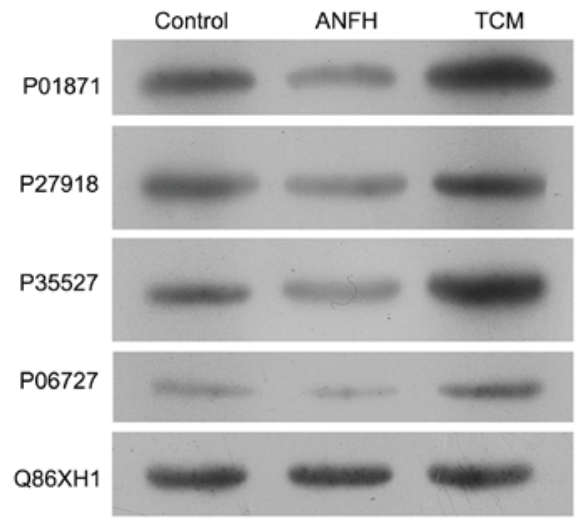

Figure 3. Western blot analysis of serum proteins in normal subjects (control) and patients with ANFH +/-TCM. ANFH, avascular necrosis of the femoral head; TCM, traditional Chinese medicine; P01871, Ig mu chain $\mathrm{C}$ region; P27918, properdin; P35527, keratin, type I cytoskeletal 9; P06727, apolipoprotein A-IV; Q86XH1, IQ and AAA domain-containing protein 1.
IGHM; P27918, properdin; P35527, KRT9; P06727, APOA4; and Q86XH1, IQCA1 (Fig. 1). The expression of these proteins was confirmed using ELISA (Fig. 2) and western blot analysis (Fig. 3). The results suggested that P01871, IGHM; P27918, properdin; P35527, KRT9; P06727, APOA4; and Q86XH1, IQCA1 were downregulated in ANFH. Following treatment with the TCM, the expression levels of all these proteins were upregulated.

\section{Discussion}

In the present study, several serum proteins were found to be downregulated in patients with $\mathrm{ANFH}$, and upregulated following treatment with TCM. These proteins were P01871, IGHM; P27918, properdin; P35527, KRT9; P06727, APOA4; and Q86XH1, IQCA1. These are potential biomarkers for the outcome of ANFH. 
Currently, the pathogenesis of ANFH primarily involves osteoporosis, vascular wall damage or compression, blood lipid disorder, high intraosseous pressure, intravascular coagulation and secondary collision (22). Secondary collision indicates that osteonecrosis of the femoral head is a multifactorial disease, and is associated with genetic susceptibility factors and exposure to specific risk factors. The occurrence of ANFH is the result of a combination of posterior acquired factors and genetic predisposing factors. A clinical study had indicated that, in patients on long-term high-dose hormone treatment, not all suffer from ANFH, with only $10 \%$ of patients affected by ANFH (23). Although there have been several clinical and basic studies regarding ANFH, it's specific pathophysiological mechanism remains to be elucidated. The application of proteomics technology in ANFH has been relatively recent, resulting in few available reports. The use of proteomics technology to examine ANFH is likely to assist in explaining the pathological and physiological mechanism of the condition.

TCM has shown to provide a curative effect in the clinical treatment of ANFH. Liu et al (24) extracted bone tissue protein samples from the femur and humerus bone of a rat osteonecrosis model for proteomics investigation. The results revealed that anticoagulating protein heavy chain II B, phospholipid hydroperoxide glutathione peroxidase and ubiquitin enzymes E2 (MW, $17 \mathrm{kD}$ ) had important regulatory roles in the occurrence of hormone-induced bone necrosis and the TCM treatment process.

High-dose immunoglobulins are important agents in the treatment of several autoimmune disorders. It has been demonstrated that high-dose immunoglobulin treatment was successful in treating a patient with steroid-induced osteonecrosis of the femoral head (25). Therefore, the increased expression of IGHM may be important in TCM treatment.

Properdin stabilizes the alternative pathway convertases, and is a positive regulator of the complement system, which amplifies the alternative pathway by extending the half-life of the C3 and C5 convertases (26). Properdin also binds to certain surfaces to provide a platform for de-novo convertase assembly (27). The serum level of properdin maybe a reliable clinical biomarker to identify patients with underlying surface alternative pathway C5 convertase dysregulation (26). The present study indicated that the alternative pathway may be involved in TCM treatment.

APOA is correlated with steroid-induced osteonecrosis of the femoral head (28). The expression of APOA4 has been reported to be lower in the serum of patients with steroid-induced osteonecrosis of the femoral head (29). This is consistent with the results of the present study, which further confirmed that the treatment was associated with the increased level of APOA4.

KRT9 is a type I intermediate filament protein (30), and IQCA1 is an important coenzyme and enzyme regulator, which interacts with ATP. In the present study, the expression of these proteins was found to be differential among the ANFH, TCM and normal subjects. The underlying mechanism remains to be elucidated.

In conclusion, the proteins, IGHM, properdin, KRT9, APOA4 and IQCA1, were identified as potential biological markers for the early clinical diagnosis of ANFH, and maybe used to develop more rapid and convenient detection strategies.

\section{Acknowledgements}

This study was financially supported by a grant from the National Natural Science Foundation of China (grant no. 81273784).

\section{References}

1. Carulli C, Nistri L, Bracco L, Giannini M and Amato MP: A steroid-induced bilateral avascular necrosis of the femoral head in an underage patient affected by multiple sclerosis. Clin Cases Miner Bone Metab 12: 257-259, 2015.

2. Kamath AF, McGraw MH and Israelite CL: Surgical management of osteonecrosis of the femoral head in patients with sickle cell disease, World J Orthop 6: 776-782, 2015.

3. Chen Y, Zeng C, Zeng H, Zhang R, Ye Z, Xing B, Hu K, Li M and Cai DZ: Comparative serum proteome expression of the steroid-induced femoral head osteonecrosis in adults. Exp Ther Med 9: 77-83, 2015.

4. Björkman A, Svensson PJ, Hillarp A, Burtscher IM, Rünow A and Benoni G: Factor $\mathrm{V}$ leiden and prothrombin gene mutation: Risk factors for osteonecrosis of the femoral head in adults. Clin Orthop Relat Re: 168-172, 2004.

5. Inoue S, Horii M, Asano T, Fujioka M, Ogura T, Shibatani M, Kim WC, Nakagawa M, Tanaka T, Hirota Y and Kubo T: Risk factors for nontraumatic osteonecrosis of the femoral head after renal transplantation. J Orthop Sci 8: 751-756, 2003.

6. Serre H and Simon L: Primary osteonecrosis of the femur head in the adult. II. Etiology and pathogenesis. Rev Rhum Mal Osteoartic 29: 536-545, 1962 (In French).

7. Li CG, Shen L, Yang YP, Xu XJ, Shuai B and Ma C: Effects of Modified Qing'e Pill () on the expression of adiponectin, bone morphogenetic protein 2 and coagulation-related factors in patients with nontraumatic osteonecrosis of the femoral head. Chin J Integr Med 23: 183-189, 2017.

8. Mont MA, Cherian JJ, Sierra RJ, Jones LC and Lieberman JR: Nontraumatic osteonecrosis of the femoral head: Where do we stand today? A ten-year update. J Bone Joint Surg Am 97: 1604-1627, 2015.

9. Xu Z, Dai X, Yao Y, Shi D, Chen D, Dai J, Teng H and Jiang Q: Higher levels of serum triglycerides were associated with postoperative deep vein thrombosis after total hip arthroplasty in patients with nontraumatic osteonecrosis of the femoral head. Int J Low Extrem Wounds 15: 41-44, 2016.

10. Seamon J, Keller T, Saleh J and Cui Q: The pathogenesis of nontraumatic osteonecrosis. Arthritis 2012: 601763, 2012.

11. Reid IR and Cornish J: Epidemiology and pathogenesis of osteonecrosis of the jaw. Nat Rev Rheumatol 8: 90-96, 2011.

12. Ikeuchi K, Hasegawa Y, Seki T, Takegami Y, Amano T and Ishiguro N: Epidemiology of nontraumatic osteonecrosis of the femoral head in Japan. Mod Rheumatol 25: 278-281, 2015.

13. Björkman A, Burtscher IM, Svensson PJ, Hillarp A, Besjakov J and Benoni G: Factor V Leiden and the prothrombin 20210A gene mutation and osteonecrosis of the knee. Arch Orthop Trauma Surg 125: 51-55, 2005.

14. Fenglei S, Gang L, Haibin W and Hao Y: Antagonism of yuanshi shengmai chenggu capsule on steroid-induced adipogenesis in rat marrow stromal cells. J Guangzhou Uni Trad Chin Med 20: 302-304, 2003.

15. Zhang YT, Geng YP, Zhou L, Lai BC, Si LS and Wang YL: Identification of proteins of human colorectal carcinoma cell line SW480 by two-dimensional electrophoresis and MALDI-TOF mass spectrometry, World J Gastroenterol 11: 4679-4684, 2005.

16. Larsen MR, Højrup P and Roepstorff P: Characterization of gel-separated glycoproteins using two-step proteolytic digestion combined with sequential microcolumns and mass spectrometry. Mol Cell Proteomics 4: 107-119, 2005.

17. Mangé A, Dimitrakopoulos L, Soosaipillai A, Coopman P, Diamandis EP and Solassol J: An integrated cell line-based discovery strategy identified follistatin and kallikrein 6 as serum biomarker candidates of breast carcinoma, J Proteomics 142: 114-121, 2016.

18. Zhang Y, Li Y, Zhang Y, Wang Z, Zhao M, Su N, Zhang T, Chen L, Wei W, Luo J, et al: Quantitative proteomics reveals membrane protein-mediated hypersaline sensitivity and adaptation in halophilic nocardiopsis xinjiangensis. J Proteome Res 15: $68-85,2016$. 
19. Narumi R and Tomonaga T: Quantitative analysis of tissue samples by combining iTRAQ isobaric labeling with selected/multiple reaction monitoring (SRM/MRM). Methods Mol Biol 1355: 85-101, 2016.

20. Zeng Y, Yao X, Chen L, Yan Z, Liu J, Zhang Y, Feng T, Wu J and Liu X: Sphingosine-1-phosphate induced epithelial-mesenchymal transition of hepatocellular carcinoma via an MMP-7/syndecan-1/TGF- $\beta$ autocrine loop. Oncotarget 7 : 63324-63337, 2016.

21. Liu JX, Yan ZP, Zhang YY, Wu J, Liu XH and Zeng Y: Hemodynamic shear stress regulates the transcriptional expression of heparan sulfate proteoglycans in human umbilical vein endothelial cell. Cell Mol Biol (Noisy-le-grand) 62: 28-34, 2016.

22. Malizos KN, Karantanas AH, Varitimidis SE, Dailiana ZH Bargiotas K and Maris T: Osteonecrosis of the femoral head: Etiology, imaging and treatment. Eur J Radiol 63: 16-28, 2007.

23. Luo YZ, He W and Sun Y: Kidney disease complicated with steroid-induced necrosis of femoral head: An analysis of 62 cases. J Guangzhou Univ Tradit Chin Med 21: 108-111, 2004 (In Chinese).

24. Liu JR, Fan YG, Wang HB, et al: Bone proteomic analysis about Chinese medicine action on rat glucocorticoid-induced model of osteonecrsis. Chinese J Trad Med Traum \& Orthop 13:4-10, 2005 (In Chinese).

25. Giesecke E, Enk A and Kerstin S: Treatment of pemphigus vulgaris with high-dose intravenous immunoglobulins in a patient with steroid-induced osteonecrosis of the femoral head. J Deuts Dermat Ges 2: 354-356, 2004.
26. Corvillo F, Bravo García-Morato M, Nozal P, Garrido S, Tortajada A, Rodríguez de Córdoba S and López-Trascasa M: Serum properdin consumption as a biomarker of C5 convertase dysregulation in C3 glomerulopathy. Clin Exp Immunol 184: 118-125, 2016.

27. Ferreira VP, Cortes $C$ and Pangburn MK: Native polymeric forms of properdin selectively bind to targets and promote activation of the alternative pathway of complement. Immunobiology 215: 932-940, 2010.

28. Cui Y, Kaisaierjiang A, Cao P, Wu ZY and Lv Q: Association of apolipoprotein A5 genetic polymorphisms with steroid-induced osteonecrosis of femoral head in a Chinese Han population. Diagn Pathol 9: 229, 2014.

29. Jiang HY, Wang SX, Li XH, Wei MT, Yang X, Chen JJ, Luan DW, Li Z, Chen YH and Weng YG: The proteomics research on relational expressed serum proteins among the recovered SARS patients complicating avascular necrosis of femoral head. Zhonghua Yu Fang Yi Xue Za Zhi 42: 522-526, 2008 (In Chinese).

30. Fu DJ, Thomson C, Lunny DP, Dopping-Hepenstal PJ, McGrath JA, Smith FJD, Irwin McLean WH and Leslie Pedrioli DM: Keratin 9 is required for the structural integrity and terminal differentiation of the palmoplantar epidermis. J Invest Dermatol 134: 754-763, 2014.

(i) (5) $९$ This work is licensed under a Creative Commons Attribution-NonCommercial-NoDerivatives 4.0 International (CC BY-NC-ND 4.0) License. 